\title{
Non-Bleeding Colonic Ulcer as Initial Manifestation of Disseminated Cryptococcosis in a Patient With Human Immunodeficiency Virus
}

Julio C. Valencia-Manrique ${ }^{1}$, Adebola Adetiloye ${ }^{1}$, Rasha Alaameri ${ }^{1}$, Jose Flores ${ }^{1}$, Gabriel Ibarra ${ }^{1}$, Yvette Achuo-Egbe ${ }^{2}$, Jennifer Harley ${ }^{2}$, Jagbir Sandhu ${ }^{3}$

1. Medicine, Metropolitan Hospital Center, New York, USA 2. Gastroenterology and Hepatology, Metropolitan Hospital Center, New York, USA 3. Pathology, Metropolitan Hospital Center, New York, USA

Corresponding author: Julio C. Valencia-Manrique, valenciamanrique@gmail.com

\begin{abstract}
Cryptococcosis is an invasive mycosis caused by Cryptococcus sp. Its presence is described closely with immunosuppressive states. Once it has reached the body, it has shown a predilection for two sites: the lungs and the central nervous system. Nonetheless, since it has hematogenous dissemination, it can colonize and yield disease at any organ. Hence, a patient will typically present with constitutional symptoms including fever, malaise, and weight loss, associated with cough, shortness of breath, chest pain, or associated headache, drowsiness, and meningeal irritation signs. We illustrate here one of the uncommon nonpulmonary non-cerebral forms of the disease of cryptococcosis, a newly diagnosed HIV/AIDS patient with a non-bleeding colon ulcer, who lacks respiratory or central nervous system (CNS)-related symptoms but endorses non-specific gastrointestinal complaints. The first evidence of the disease was the elevated cryptococcal antigen (CrAg). The direct visualization of the spores in the biopsy confirmed the infection.
\end{abstract}

Categories: Internal Medicine, Gastroenterology, Infectious Disease Keywords: cryptococcus, hiv aids, colon ulcer, grubii, disseminated

\section{Introduction}

Cryptococcosis is an invasive fungal infection that is highly prevalent among immunocompromised individuals. It is known to cause approximately $15 \%$ of AIDS-related mortality worldwide [1]. Cryptococcus neoformans is the principal member of the genus. The portal of entry into the organism is primarily the lungs. It colonizes the alveoli and can remain dormant or has a hematogenous spread and potentially invades any organ. Meningitis and meningoencephalitis are the most frequent manifestations of cryptococcosis. Other less affected systems include the gastrointestinal tract, which has been described occasionally in the literature. We present a case of cryptococcosis initially manifested as a non-bleeding sigmoid ulcer in a patient with a previously unknown HIV status.

Review began 08/08/2021 Review ended 08/12/2021 Published 08/19/2021

\section{(c) Copyright 2021} Valencia-Manrique et al. This is an open access article distributed under the terms of the Creative Commons Attribution License CC-BY 4.0., which permits unrestricted use, distribution, and reproduction in any medium, provided the original author and source are credited.

\section{Case Presentation}

A 54-year-old female with a history of hypertension, dyslipidemia, and chronic anemia underwent a colonoscopy and esophagogastroduodenoscopy (EGD) as part of the investigation of concomitant weight loss and retrosternal pain. The studies disclosed a 7mm-ulcer (Figure 1) in the sigmoid colon, whereas the EGD revealed esophageal lesions typical for candidiasis. She was started on oral fluconazole and sent for HIV testing. The patient returned two days later for the results of the test. Upon disclosing the positive results of the HIV test, she suffered a pre-syncopal episode which led her to the emergency room. On further inquiry, she mentioned feeling fatigue, abdominal pain, and mild dysphagia for several months. The physical exam was positive for oral thrush and mild diffuse abdominal tenderness to deep palpation. Meanwhile, the initial blood tests were remarkable for anemia (Hemoglobin $11.5 \mathrm{~g} / \mathrm{dL}$ ) and leukopenia (leukocytes 2440/mcL). EKG was normal. The patient remained under observation for a vasovagal nearsyncope and newly diagnosed HIV infection. Additional workup to investigate the sigmoid ulcer was negative for varicella, toxoplasma, histoplasmosis, and T. pallidum. Cytomegalovirus was positive only for the IgG antibody. CD4-count was 19 cells $/ \mathrm{m}^{3}$ and the viral load $1.3 \times 106$ copies/ml. CT abdomen was normal. 


\section{Cureus}

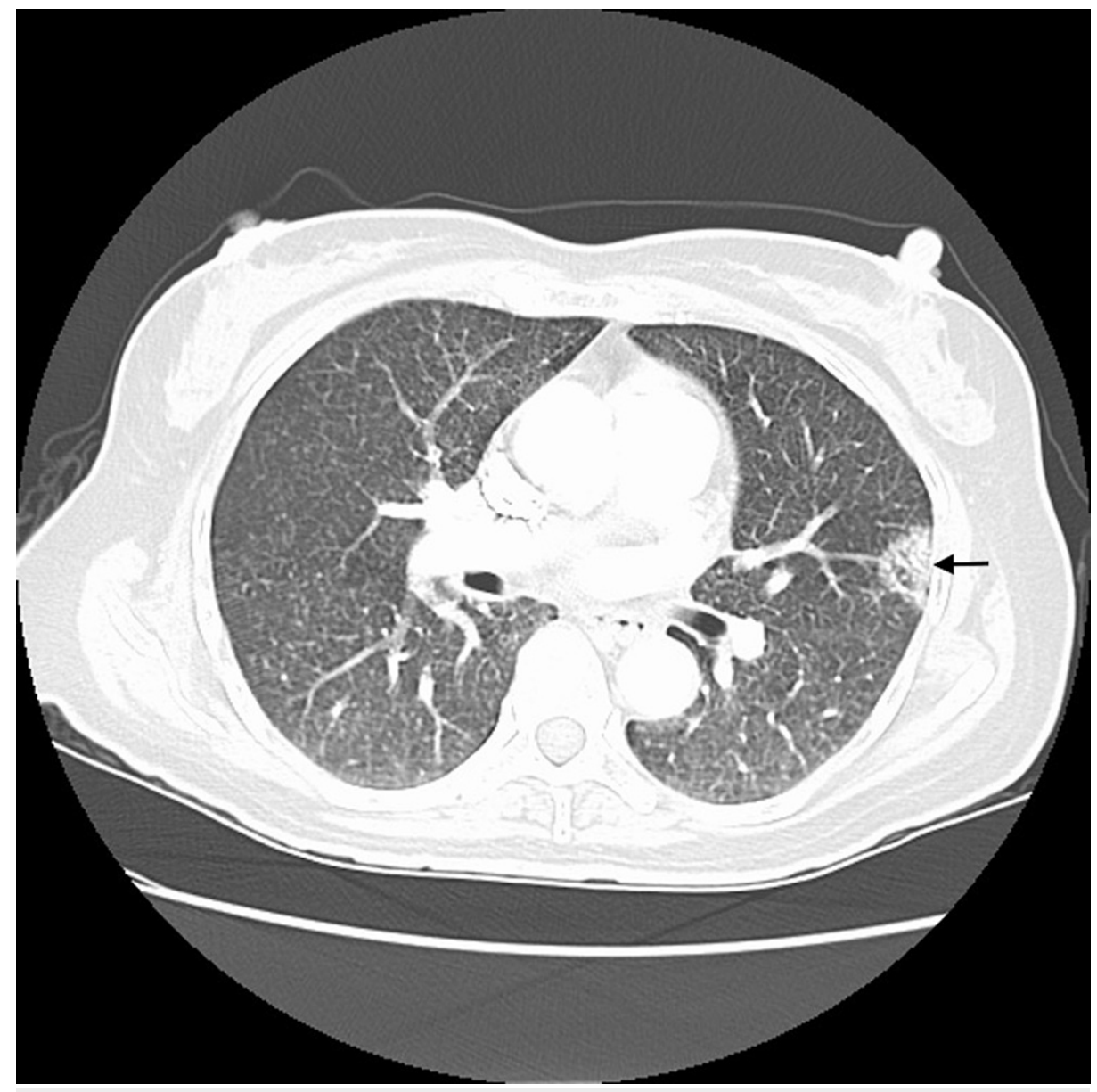

FIGURE 1: Computerized tomography of the chest without contrast, showing a mass abutting pleura in the apico-posterior segment of the left upper lobe (black arrow). Bilateral tiny reticulo-nodular densities can be seen as well.

The evidence of diffuse bilateral reticulonodular densities in the chest X-ray led to testing for methicillinresistant staphylococcus aureus/methicillin-sensitive staphylococcus aureus (MRSA/MSSA), respiratory viruses, aspergillosis, legionella, tuberculosis, and fungal infections. All the above-mentioned tests were negative, but cryptococcal antigen (CrAg) was highly positive >1:2560 dilution. P. jirovecii test was not reported due to a processing error. CT chest confirmed the reticulonodular densities and also noted a mass in the left upper lobe (Figure 1). It was considered a possible cryptococcoma.

One day after admission, the colon biopsy unveiled chronic inflammatory cells, multinucleated giant cells, and spores of cryptococcus (Figure 2), and the esophageal biopsies confirmed candida esophagitis. In light of the confirmed disseminated cryptococcal infection (elevated $\mathrm{CrAg}$, positive colon biopsy, and possible cryptococcoma), the patient was started on amphotericin $275 \mathrm{mg}$ daily alongside fluconazole $800 \mathrm{mg}$ in addition to prophylaxis for opportunistic infections. 

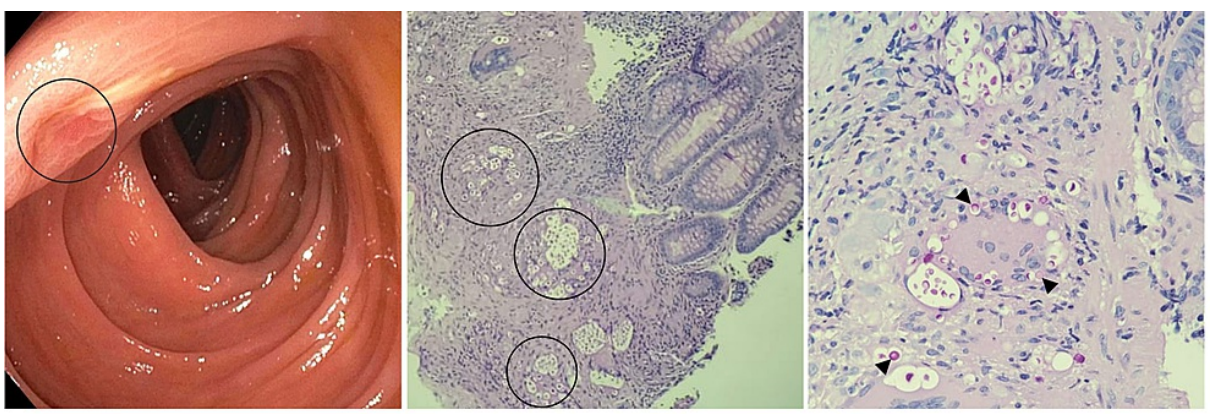

FIGURE 2: (A) Colonoscopy, a small non-bleeding ulcer observed in the left upper quadrant (black circle). (B \& C) Biopsy 40x and 100x, PAS demonstrates the presence of the encapsulated, round-shaped spores (arrowheads) consistent with cryptococcosis, some of them distributed in cluster (black circles).

PAS: Periodic acid-Schiff satin

In the following days, the patient started complaining of mild to moderate headaches. It warranted a lumbar puncture to evaluate a possible central nervous system (CNS) compromise. The opening pressure was 28 $\mathrm{cmH} 2 \mathrm{O}$. The fluid was clear, colorless, low in glucose $(22 \mathrm{mg} / \mathrm{dL})$, and $2 \%$ polymorphonuclear cells. The venereal disease research laboratory (VDRL) and the gram stain were negative. Moreover, India ink, meningitis panel-polymerase chain reaction (PCR), and cerebrospinal fluid (CSF) cultures were positive for cryptococcus. These findings confirmed cryptococcal meningitis.

During the hospitalization, she underwent serial imaging of the head to evaluate for elevated intracranial pressure. In addition, she got three more lumbar punctures (LPs), the last showed a normal opening pressure $(8 \mathrm{~cm}-\mathrm{H} 2 \mathrm{O})$, and the CSF culture did not grow any yeast. After six weeks of induction therapy, the patient was transitioned to consolidation therapy and started antiretroviral therapy (ART). The initial symptoms resolved. The patient left the hospital with a plan to follow up in the virology clinic.

\section{Discussion}

Cryptococci are encapsulated yeasts of worldwide distribution. The genus is composed of more than 35 species, among which only two, C. neoformans and C. gattii, have been attributed pathogenic properties. C. neoformans, principally the variety grubii, is responsible for most of the cases of cryptococcosis in immunosuppressed patients, whereas C. gattii has been associated mainly with immunocompetent subjects. C. neoformans proliferates in soil rich in avian droppings. Humans may get infected after being exposed to contaminated vegetation. Inhalation of the basidiospore forms represents the primary route of transmission. Interestingly, the yeast holds a predilection for the central nervous system, which favors brain colonization. Consequently, the presence of respiratory symptoms (accredited to the lungs being the initial site of colonization) or neurologic symptoms (explained by neurotropism) configures the most common manifestation of cryptococcosis.

However once in the body, the yeast can widely disseminate, and be detectable at any organ [2]. Indeed, the first clinical identification of the disease was in a tibia with osteomyelitis. Gastrointestinal cryptococcosis (GIC) has been occasionally reported. Osawa and Singh [3], after a literature review, found 30 cases of GIC reported from 1951 to 2008. Most of the cases of GIC outlines an underlying impaired immune response. HIV/AIDS represents the leading cause of immunosuppression, yet conditions like intestinal lymphangiectasia, immunosuppressive therapy, primary biliary cirrhosis, and malignancies account as alternative sources of weakened immunity. In the settings of HIV/AIDS, gastrointestinal involvement correlates to low CD4 count $\left(<50-100 \mathrm{cell} / \mathrm{mm}^{3}\right)$ and typically denotes severe dissemination [3], warranting CNS evaluation regardless of the presence of neurologic symptoms.

In terms of symptomatology, literature refers to GIC as a "mimicker" manifesting as abdominal pain, intestinal mass, mucosal inflammation, mucosal ulcer (as seen in this case), or even isolated iron deficiency anemia. In addition, there is a subgroup of patients which lacks gastrointestinal manifestation, as illustrated by Dromer et al. [4] in a nine-year nationwide epidemiological study in France, and by Washington et al. [5], who determined that $33 \%$ of autopsies of known cases of pulmonary and disseminated cryptococcosis were also positive for GIC.

We get to diagnose GIC via endoscopic biopsy, detecting the yeast in the gastrointestinal submucosa. Feces culture is also helpful, but its sensitivity is low. Elevated CrAg is an additional marker that should prompt 
further testing to evaluate dissemination and CNS compromise [6]. Amphotericin B is the cornerstone of the treatment in the disseminated form, along with flucytosine or fluconazole.

\section{Conclusions}

We conclude that even though GIC is not a usual presentation of cryptococcosis, it should always be considered in patients with any condition hindering the immune system (particularly HIV/AIDS patients) who develop gastrointestinal symptoms and tested negative for more frequent etiologies. GIC should also raise suspicion for dissemination and lead us to evaluate for possible CNS involvement. Elevated CrAg is an additional marker of dissemination and CNS compromise.

\section{Additional Information}

\section{Disclosures}

Human subjects: Consent was obtained or waived by all participants in this study. Conflicts of interest: In compliance with the ICMJE uniform disclosure form, all authors declare the following: Payment/services info: All authors have declared that no financial support was received from any organization for the submitted work. Financial relationships: All authors have declared that they have no financial relationships at present or within the previous three years with any organizations that might have an interest in the submitted work. Other relationships: All authors have declared that there are no other relationships or activities that could appear to have influenced the submitted work.

\section{References}

1. Rajasingham R, Smith RM, Park BJ, et al.: Global burden of disease of HIV-associated cryptococcal meningitis: an updated analysis. Lancet Infect Dis. 2017, 17:873-881. 10.1016/S1473-3099(17)30243-8

2. Maziarz EK, Perfect JR: Cryptococcosis. Infect Dis Clin North Am. 2016, 30:179-206. 10.1016/j.idc.2015.10.006

3. Osawa R, Singh N: Colitis as a manifestation of infliximab-associated disseminated cryptococcosis . Int J Infect Dis. 2010, 14:e436-e440. 10.1016/j.ijid.2009.05.019

4. Dromer F, Mathoulin S, Dupont B, Laporte A: Epidemiology of cryptococcosis in France: a 9-year survey (1985-1993). Clin Infect Dis. 1996, 23:82-90. 10.1093/clinids/23.1.82

5. Washington K, Gottfried MR, Wilson ML: Gastrointestinal cryptococcosis. Mod Pathol. 1991, 4:707-711.

6. Perfect JR, Dismukes WE, Dromer F, et al.: Clinical practice guidelines for the management of cryptococcal disease: 2010 update by the Infectious Diseases Society of America. Clin Infect Dis. 2010, 50:291-322. $10.1086 / 649858$ 